\title{
Milliliter per Minute per Meter Squared
}

National Cancer Institute

\section{Source}

National Cancer Institute. Milliliter per Minute per Meter Squared. NCI Thesaurus. Code C120803.

A unit of concentration equal to milliliter per minute divided by meter squared. 